\title{
Movement Initiation-Locked Activity of the Anterior Putamen Predicts Future Movement Instability in Periodic Bimanual Movement
}

\author{
Yu Aramaki, ${ }^{1,2,3}$ Masahiko Haruno, ${ }^{3}$ Rieko Osu, ${ }^{4}$ and Norihiro Sadato ${ }^{5}$ \\ ${ }^{1}$ Laboratory for Exercise Physiology and Biomechanics, School of Health and Sport Sciences, Chukyo University, Kaizu-cho, Toyota, Aichi 470-0393, Japan, \\ ${ }^{2}$ Center for Fostering Young and Innovative Researchers, Nagoya Institute of Technology, Gokiso-cho, Showa-ku, Nagoya, Aichi 466-8555, Japan, ${ }^{3}$ National \\ Institute of Information and Communications Technology, Nishi-ku, Kobe, Hyogo 651-2492, Japan, ${ }^{4}$ ATR Computational Neuroscience Laboratories, \\ Keihanna Science City, Kyoto 619-0288, Japan, and 5 Department of Cerebral Research, National Institute for Physiological Sciences, Myodaijicho, Okazaki, \\ Aichi 444-8585, Japan
}

In periodic bimanual movements, anti-phase-coordinated patterns often change into in-phase patterns suddenly and involuntarily. Because behavior in the initial period of a sequence of cycles often does not show any obvious errors, it is difficult to predict subsequent movement errors in the later period of the cyclical sequence. Here, we evaluated performance in the later period of the cyclical sequence of bimanual periodic movements using human brain activity measured with functional magnetic resonance imaging as well as using initial movement features. Eighteen subjects performed a $30 \mathrm{~s}$ bimanual finger-tapping task. We calculated differences in initiationlocked transient brain activity between antiphase and in-phase tapping conditions. Correlation analysis revealed that the difference in the anterior putamen activity during antiphase compared within-phase tapping conditions was strongly correlated with future instability as measured by the mean absolute deviation of the left-hand intertap interval during antiphase movements relative to in-phase movements $(r=0.81)$. Among the initial movement features we measured, only the number of taps to establish the antiphase movement pattern exhibited a significant correlation. However, the correlation efficient of 0.60 was not high enough to predict the characteristics of subsequent movement. There was no significant correlation between putamen activity and initial movement features. It is likely that initiating unskilled difficult movements requires increased anterior putamen activity, and this activity increase may facilitate the initiation of movement via the basal ganglia-thalamocortical circuit. Our results suggest that initiation-locked transient activity of the anterior putamen can be used to predict future motor performance.

\section{Introduction}

It is currently unclear whether brain activity signals can be used to predict future errors in ongoing periodic movements. Several recent studies using functional magnetic resonance imaging (fMRI) reported that brain activity can predict discrete task performance, including simple reaction time (RT) (Weissman et al., 2006) and flanker tasks (Eichele et al., 2008). However, no studies have successfully predicted future errors in ongoing periodic movements.

\footnotetext{
Received Aug. 26, 2010; revised May 13, 2011; accepted May 19, 2011.

Author contributions: Y.A. designed research; Y.A. performed research; Y.A. contributed unpublished reagents/ analytic tools; Y.A. analyzed data; Y.A., M.H., R.O., and N.S. wrote the paper.

This work was supported by KAKENHI (Grant 22700596 to Y.A.; Grant 22300139 to M.H.; and Grant 21220005 to N.S.), the Cooperative Study Program of National Institute for Physiological Sciences (Y.A. and N.S.), Precursory Research for Embryonic Science and Technology, the Japan Science and Technology Agency (M.H.), the Funding Program for Next Generation World-Leading Researchers (R.0.), and the Strategic Research Program for Brain Sciences (Ministry of Education, Culture, Sports, Science, and Technology in Japan). We grateful to Mr. Toshinori Yoshioka (Computational Neuroscience Laboratories Technical Support Group in Advanced Telecommunication Research Institute) for technical support.

This article is freely available online through the J Neurosci Open Choice option.

Correspondence should be addressed to Dr. Yu Aramaki, Laboratory for Exercise Physiology and Biomechanics, School of Health and Sport Sciences, Chukyo University, 101 Tokodachi, Kaizu-cho, Toyota, Aichi 470-0393, Japan. E-mail:yu_armk@sass.chukyo-u.ac.jp.

DOI:10.1523/JNEUROSCI.4473-10.2011

Copyright $\odot 2011$ the authors $\quad 0270-6474 / 11 / 319819-05 \$ 15.00 / 0$
}

The periodic bimanual coordination task comprises two typical modes with different levels of stability, including the in-phase and antiphase modes (Yamanishi et al., 1980; Kelso, 1984), making it ideal for studying sudden and unpredictable human movement error. The antiphase mode, which requires the simultaneous activation of nonhomologous muscle groups [i.e., "bimanual parallel" (BP)] (see Fig. 1 $a$, bottom), is less stable than the in-phase mode, which requires the activation of homologous muscle groups [i.e., "bimanual mirror" (BM)] (see Fig. 1a, top) (Kelso, 1984). For the same movement frequency, antiphase movement is typically more variable than in-phase movement (Kelso, 1984). This feature maps onto a theoretical model (Haken et al., 1985; Kelso, 2010). The difference in stability between the two modes has been explained in terms of the tendency toward coactivation of homologous muscle groups (Kelso, 1984) or a preference for perceptual symmetry (Mechsner et al., 2001). The difference in stability between the two modes can induce spontaneous phase transitions from the antiphase to the in-phase mode (Kelso, 1984) even when attempting to maintain an antiphase movement pattern, although the intentional component can stabilize an otherwise unstable antiphase pattern to some degree (Scholz and Kelso, 1990; Kelso, 1995; De Luca et al., 2010). In 
addition to the intentional component, the stability of antiphase movements also depends on learning (Temprado et al., 2002), perceptual bias (Mechsner et al., 2001), attention (Monno et al., 2000), and neural connections (Kennerley et al., 2002; Kagerer et al., 2003). Because the probability of these sudden errors differs between subjects (see Fig. $1 b$ ), it appears difficult to predict future movement performance from the initial period of sequence of movement cycles. In the present study, we investigated whether future performance could be predicted from initiation-locked brain activity, as well as from several initial movement features.

Several lines of evidence suggest that the basal ganglia, which include the putamen, are likely to be involved in predicting future motor performance. Putamen neurons have been reported to exhibit transient firing during the movement-initiation phase, and this neuronal activity may be related to motor programming or general attention (Kimura, 1990; Romo et al., 1992). Previous bimanual coordination studies reported that initiating an antiphase pattern required greater transient putamen activity than initiating an in-phase pattern (Kraft et al., 2007; Aramaki et al., 2010). However, the relationship between transient putamen activity during the initiation phase and subsequent future behavioral performance remains unclear.

We hypothesized that the magnitude of the difference in transient putamen activity during the initiation phase between modes would correlate with future movement performance, and predict the future motor performance of individual subjects.

\section{Materials and Methods}

We reused the data of Aramaki et al. (2010), and added one subject. While Aramaki et al. (2010) focused on the difference in brain activity between the sum of right and left unimanual movement and bimanual movement at the group level, the present study focused on the relationship between behavioral performance and brain activity at the individual level.

\section{Subjects}

Eighteen healthy volunteers (age range, 25-38 years; mean age \pm SD, $29.7 \pm 4.01$ years; 10 males, eight females) participated in the present study. All subjects were right handed (Edinburgh handedness inventory, $91 \pm 14)$. None of the subjects had a history of psychiatric or neurological illness. Three subjects were university undergraduates, and the other 15 subjects were postgraduates. Nine subjects had learned the piano for several years in their childhood. All subjects work with computers on a daily basis, so the high-frequency tapping task was not difficult, particularly in the BM condition.

The study protocol was approved by the Ethics Committee of the National Institute of Physiological Sciences, Japan. All subjects provided written informed consent before participating in the study.

\section{Experimental task}

The subjects performed a $30 \mathrm{~s}$ periodic, bimanual, four-finger tapping task (Kelso, 1995; p. 46) in the BM and BP modes (Fig. 1a). We defined the BM mode as symmetrical or in-phase movement of homologous effectors, involving the synchronous tapping of both index fingers alternating periodically with the synchronous tapping of both middle fingers:

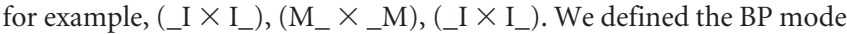
as asymmetrical or antiphase movement resulting from the simultaneous activity of nonhomologous effectors, involving the synchronous tapping of the left middle and the right index fingers alternating periodically with synchronous tapping of the left index and the right middle fingers: for example, $\left(M_{-} \times I_{-}\right),\left(\_I \times \_M\right),\left(M_{-} \times I_{-}\right)$. Subjects were instructed to restart as quickly as possible if the tapping stopped or switched to another mode.

Auditory cues were presented at $3 \mathrm{~Hz}$ to help subjects maintain a constant tapping frequency. We previously found that a movement frequency of $3 \mathrm{~Hz}$ is appropriate for observing individual differences in this task (Aramaki et al., 2006). Two USB MRI-compatible 10-key a
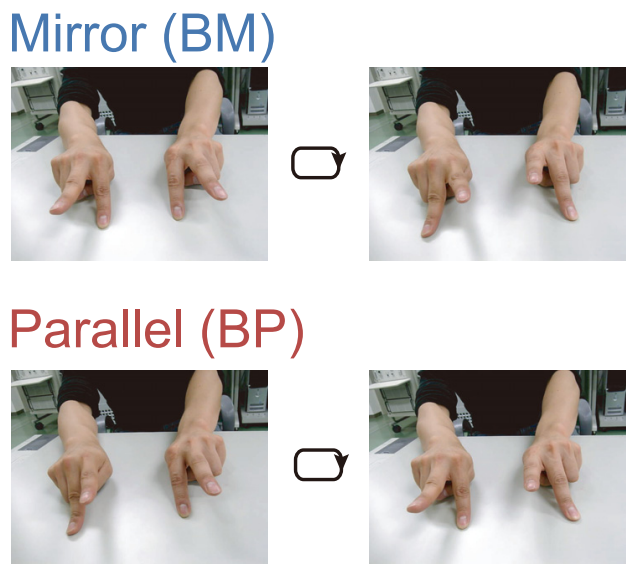

b
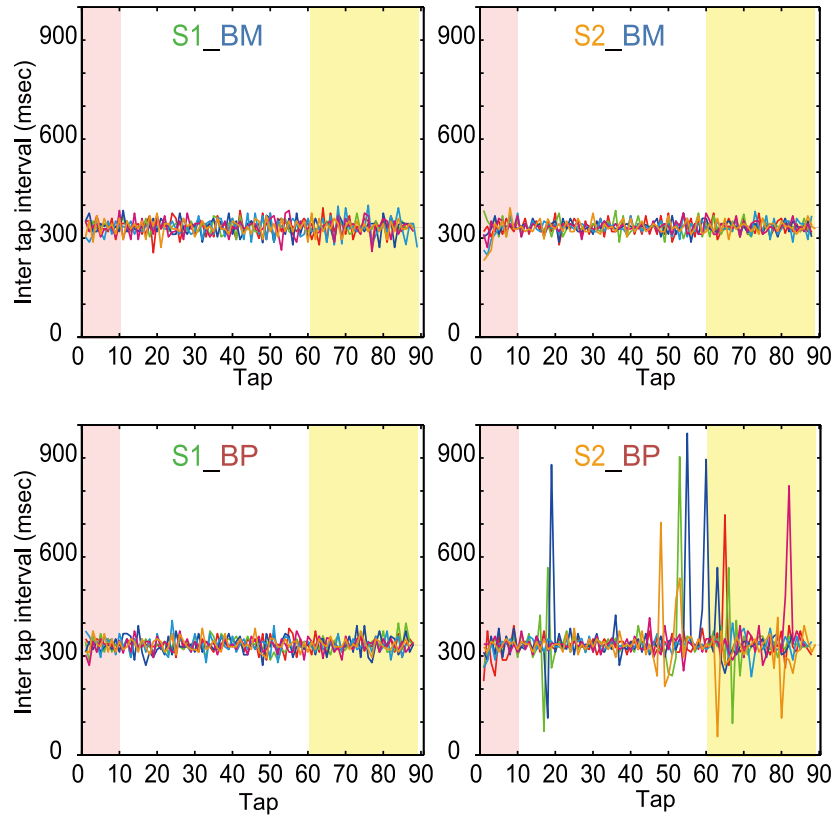

Figure 1. $\quad \boldsymbol{a}$, Bimanual tasks used in the present study. In the BP mode, subjects performed synchronous tapping of the left middle and the right index fingers, periodically alternating the synchronous tapping of the left index and right middle fingers. In the BM mode, the synchronous tapping of both index fingers alternated periodically with the synchronous tapping of both middle fingers. $\boldsymbol{b}$, Time course of the ITI of a skilled subject (S1, left) and an unskilled subject (S2, right) in the BM (top) and the BP (bottom) modes. All six trials (red, blue, magenta, cyan, green, and orange lines) for each mode during fMRI are superimposed. Pink zone indicates the initial movement phase (first 10 taps). The yellow zone indicates the future movement phase (after tap 60). The dashed line at 333 ms corresponds to the target ITI.

pads (TK-UYGT, ELECOM) were used to record finger taps. For righthanded finger taps, the keys " 1 " (for the index finger) and " 3 " (for the middle finger) of a 10-key pad were used. For the left hand, the keys " 7 " (for the index finger) and "9" (for the middle finger) of another 10-key pad were used. For each tapping mode, the fMRI session consisted of four rest epochs alternating with three $30 \mathrm{~s}$ task epochs. During each session, the name of the condition was visually presented once before the session began. Subjects were required to fix their gaze on the crosshair presented on the screen, so that they could not see their hands. The fixation point turned green ("go") and red ("stop") every $30 \mathrm{~s}$, and the auditory cue was provided continuously throughout the scanning session. Subjects performed two sessions per condition, and the order of conditions was counterbalanced across subjects. Presentation software (Neurobehavioral Systems) was used to provide auditory cues and record the timing of the key presses at $1000 \mathrm{~Hz}$. To minimize head motion, we used tight but 
comfortable foam padding placed around the subjects' heads. An LCD projector (DLA-M200L, Victor) located outside and behind the scanner projected the crosshair through another waveguide to a translucent screen, which the subjects viewed via a mirror attached to the head coil of the MRI scanner.

\section{Data analysis}

Unfortunately, we were unable to obtain right-hand tapping data from five subjects due to mechanical failure of the right-hand keypad. As such, we have partial data (13 subjects) for right-handed tapping. However, nondominant left-hand movements have been reported to fluctuate more in the BP mode than those of the dominant right hand (Semjen et al., 1995; Aramaki et al., 2006). Thus, we calculated performance indices using left-hand tapping data for all 18 subjects and used it in the correlation analysis with brain activity. Supplementarily, however, we analyzed the right-hand tapping case using 13 subjects.

\section{Behavioral performance data analysis}

Index of future performance. We used taps after the 60th tap $(\sim 10 \mathrm{~s})$ as a measure of future performance, because it allowed a sufficient time difference between this period and the brain activity associated with movement initiation. If a phase transition occurs in this period, and subjects stop movement briefly to restart the task, the mean deviation of the intertap interval (ITI) from the target ITI $(333 \mathrm{~ms})$ would be expected to increase.

To evaluate performance, we first calculated the mean deviation of the ITI from the target ITI $(333 \mathrm{~ms})$ in each condition. We then calculated the ratio of the mean absolute ITI deviation of taps after the 60th tap in the $\mathrm{BP}$ mode to deviation in the $\mathrm{BM}$ mode. This ratio was used as an index of future motor performance.

Indices of initial performance. We calculated the following three indices of initial performance. (1) The BP/BM ratio of the mean absolute ITI deviation of taps during the first 10 taps was calculated. Similar to the future performance index, we calculated the ratio of mean absolute ITI deviation of the first 10 taps in the BP mode to deviation in the BM mode. (2) The number of taps to establish a BP pattern was calculated. We evaluated this by the number of taps required to attain a given level of performance, since unskilled subjects might show longer times. We first set three intertap interval performance levels: $333 \pm 67 \mathrm{~ms}$ (20\% deviations); $333 \pm 83 \mathrm{~ms}$ ( $25 \%$ deviations); and $330 \pm 100 \mathrm{~ms}$ (30\% deviations). When subjects maintained four consecutive taps within the range, we recorded the ordinal number of the first tap among four consecutive taps as the number of taps required to attain a given performance level. (3) BP/BM RT ratio was calculated. We calculated RTs, defined as the period from cue onset to first tap. There were six RTs in each condition, because we ran two sessions of three task epochs in each session. We then calculated the ratio of mean RT in the BP mode to mean RT in the BM mode.

\section{fMRI data acquisition and analysis}

A time course series of 71 volumes was acquired in each session using $\mathrm{T} 2{ }^{\star}$-weighted gradient echoplanar imaging (EPI) sequences (repetition time, $3000 \mathrm{~ms}$; echo time, $30 \mathrm{~ms}$; flip angle, $85^{\circ}$; field of view, $192 \mathrm{~mm}$; voxel size, $3.0 \times 3.0 \times 3.0 \mathrm{~mm}$; slice number, 44 axial slices) with a 3.0 tesla MRI scanner (Allegra, Siemens). T1-weighted images were obtained with location variables identical to those of the EPI sequences. In addition, a high-resolution structural image was acquired using a magnetizationprepared rapid acquisition in gradient echo sequence.

SPM99 software (Wellcome Department of Cognitive Neurology) was used for image processing and analysis. For each subject, after discarding the first six images of each session, the remaining 65 images were realigned to correct for head motion, spatially normalized to the T1 MNI template, mediated by the 3D high-resolution T1 image and the T1weighted image with locations identical to the fMRI data, and resampled, resulting in $2 \times 2 \times 2 \mathrm{~mm}$ voxels, and smoothed spatially with an isotropic Gaussian kernel of 6 mm FWHM.

To detect movement initiation-related brain activity and movement continuation-related activity, we first decomposed brain activity into initiation-related and continuation-related activity using two regressors included in the general linear model (Aramaki et al., 2010). Initiation of movement was expressed as a delta function, so had no duration. Continuation of movement was expressed as a boxcar function with a duration of $30 \mathrm{~s}$. Each neuronal model was convolved with a hemodynamic response function.

Since we sought to identify the relationship between initiation-related brain activity and future motor performance, we generated $\mathrm{BP}>\mathrm{BM}$ contrast maps of initiation-related activity for all subjects. These maps were then used in a simple correlation analysis with the future motor performance index of each subject, focusing on the anatomical region of interest (ROI; in this case, the striatum). We used a threshold of $p<0.05$, corrected for multiple comparisons at the cluster level.

To view the qualitative temporal characteristics of the data, we extracted the time course of the mean signal intensity in the ROI from the smoothed images of each subject using MarsBaR (Brett et al., 2002) (http://marsbar.sourceforge.net/). We used "multi_color" software (http://www.cns.atr.jp/multi_color/) to display functional brain maps.

\section{Results}

First, we found a significant correlation between initiationlocked putamen activity and future performance. The striatum, including the putamen, was chosen as the anatomical ROI (Fig. $2 a$ ). As predicted, the difference between initial transient activity of the anterior putamen in the BP and BM modes correlated with future motor performance, measured by the ratio of mean absolute ITI deviation for left-hand tapping after 60 taps in the BP mode to that in the BM mode (left side: local maximum $=-26$, $2,-6$; cluster size $=132 ; p<0.001$ corrected for multiple comparisons at the cluster level; right side: local maximum $=24,8$, -6 ; cluster size $=55 ; p=0.004$ corrected for multiple comparisons at the cluster level). Figure $2 b$ shows the difference in initiation-related activity in all subjects between BP and BM modes in the left anterior putamen (cluster size $=132$ ), with the ratio of the mean absolute ITI deviation of left-hand tapping after 60 taps in BP divided by that in BM $[r=0.814, p<0.001$ for the left side (Fig. 2b, Table 1); $r=0.765, p<0.001$ for the right side (Table 1)]. Similarly, left putamen activity was significantly correlated with the ratio of the mean absolute ITI deviation of the right hand tapping after 60 taps in $\mathrm{BP}$ divided by that in BM ( $r=$ $0.649, p=0.016$ ) (Table 1 ).

Figure $2 c$ shows the time course of the blood oxygen leveldependent signal in the left anterior putamen (cluster size $=132$ ) for S1 (skilled, left) and S2 (unskilled, right). S2 showed a significant difference in the amplitude of the peak transient putamen activity between the $\mathrm{BP}(1.01 \pm 0.07)$ and BM modes $(0.52 \pm$ $0.12, p=0.008)$ (Fig. $2 c$, right). In contrast, S1 showed no difference in peak transient activity between the two modes ( $\mathrm{BP}=$ $0.75 \pm 0.13 ; \mathrm{BM}=0.59 \pm 0.06 ; p=0.286$ ) (Fig. $2 c$, left). Because the transient, initiation-related activity was not directly associated with performance after 60 taps $(\sim 10 \mathrm{~s})$, the results suggest that transient activity of the anterior putamen can predict future motor performance.

Second, we found a significant correlation between one of our initial behavioral indices and future performance (i.e., between the number of taps needed to attain the $\pm 25 \%$ deviation level in ITI performance and the BP/BM ratio of the mean absolute ITI deviation of taps after the 60th tap for left-hand tapping data) (Table 1). The other initial behavioral indices were not significantly correlated with future performance (Table 1).

None of the initial movement features we examined were significantly correlated with initiation-locked putamen activity (Table 1), though the correlation between left putamen activity and the number of taps to establish a performance level of $25 \%$ deviation for left-hand tapping data approached statistical significance ( $p=0.107$ for left putamen; $p=0.101$ for right putamen). 


\section{a}

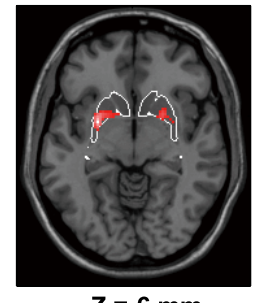

$\mathrm{Z}=-6 \mathrm{~mm}$

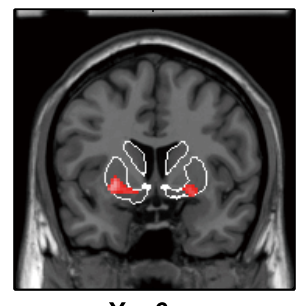

$Y=6 \mathrm{~mm}$

b

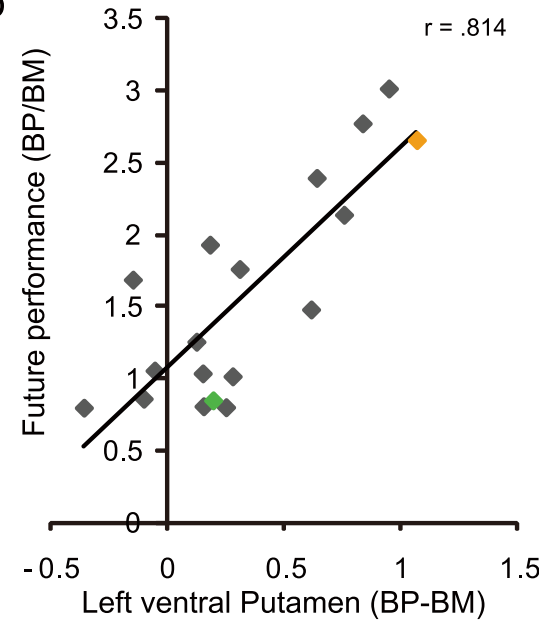

C
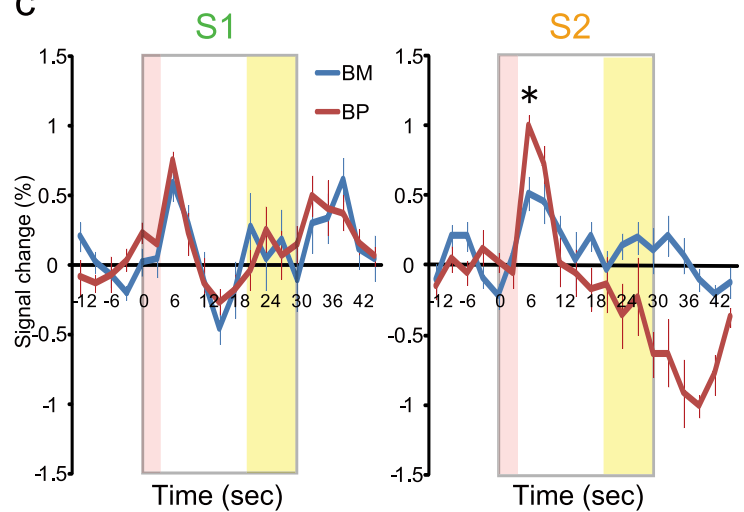

Figure 2. $\quad \boldsymbol{a}$, Results of the simple correlation analysis in the bilateral anterior putamen. The white contour is the predefined anatomical ROl (the striatum). $\boldsymbol{b}, \mathrm{A}$ plot of the data from all subjects showing that the individual parameter estimation of $B P>B M$ for the cluster representing the left anterior putamen (horizontal) was strongly correlated with future motor performance (vertical). The green point corresponds to S1. The orange point corresponds to S2. C, Time course plot of the left anterior putamen in Figure $2 a$ (cluster size $=132$ in the left side; local $\max =-26,2,-6$ ) in the BM (blue) mode and the BP (red) mode for S1 (skilled, left) and S2 (unskilled, right). The gray frame corresponds to a 30 s movement epoch. The error bars show the SE. ${ }^{*} p<0.05$.

\section{Discussion}

The current results demonstrate that initiation-related transient brain activity is useful for predicting the future stability of periodic bimanual movement. The relationship between the stability
Table 1. Correlation coefficients between behavioral and behavioral/brain indices

\begin{tabular}{lccc}
\hline & dev_after60 & putamen_L & putamen_R \\
\hline Left hand $(n=18)$ & & & \\
dev_after60 & 1 & $0.814\left(p<0.001^{*}\right)$ & $0.765\left(p<0.001^{*}\right)$ \\
dev_first10 & $0.194(p=0.441)$ & $0.187(p=0.456)$ & $0.158(p=0.532)$ \\
Number of taps ( $\pm 20 \%)$ & $0.097(p=0.70)$ & $0.081(p=0.750)$ & $0.006(p=0.982)$ \\
Number of taps ( $\pm 25 \%)$ & $0.600\left(p=0.009^{*}\right)$ & $0.393(p=0.107)$ & $0.399(p=0.101)$ \\
Number of taps ( $\pm 30 \%)$ & $0.453(p=0.059)$ & $0.214(p=0.395)$ & $0.199(p=0.428)$ \\
RT & $-0.252(p=0.314)$ & $0.01(p=0.969)$ & $-0.21(p=0.403)$ \\
Right hand $(n=13)$ & & & \\
dev_after60 & 1 & $0.649\left(p=0.016^{*}\right)$ & $0.463(p=0.111)$ \\
dev_first10 & $0.482(p=0.095)$ & $0.425(p=0.147)$ & $0.217(p=0.477)$ \\
Number of taps ( $\pm 20 \%)$ & $-0.22(p=0.471)$ & $-0.240(p=0.429)$ & $-0.385(p=0.194)$ \\
Number of taps $( \pm 25 \%)$ & $-0.166(p=0.589)$ & $-0.392(p=0.185)$ & $-0.389(p=0.189)$ \\
Number of taps $( \pm 30 \%)$ & $-0.166(p=0.587)$ & $-0.373(p=0.209)$ & $-3.63(p=0.222)$ \\
RT & $-0.141(p=0.645)$ & $0.003(p=0.993)$ & $-0.246(p=0.417)$ \\
\hline
\end{tabular}

dev after60, BP/BM ratio of the mean absolute ITI deviation of taps after the 60th tap; dev first10, BP/BM ratio of the mean absolute ITI deviation of taps during first 10 taps; number of taps, the number of taps needed to attain a given level ( $\pm \%$ deviation) of inter-tap-interval performance; $\mathrm{RT}, \mathrm{BP} / \mathrm{BM}$ ratio of reaction time. ${ }^{*} p<0.05$.

of periodic bimanual movement and brain activity has previously been studied by comparing epoch-related brain activity during in-phase and antiphase movements (Sadato et al., 1997; MeyerLindenberg et al., 2002; Aramaki et al., 2006, 2010; Kraft et al., 2007; Müller et al., 2009). However, relatively few studies have focused on transient brain activity during the movement initiation or pattern-switching phases (Aramaki et al., 2006, 2010; Kraft et al., 2007; De Luca et al., 2010). Our findings are consistent with previous studies reporting greater transient brain activity in the putamen during antiphase movements relative to in-phase movements (Kraft et al., 2007; Aramaki et al., 2010). In addition, we found that initiation-related activity in the putamen significantly predicted future motor performance. Empirically, we can consider a factor to be "predictable" when the coefficient of determination $\left(R^{2}\right)$ is $>0.5$, meaning that the correlation coefficient is $>0.707$. In this study, sufficiently high correlation coefficients were observed only between future motor performance and left putamen activity $(r=0.814)$. Thus, we conclude that brain activity can predict future motor performance. On the other hand, among the indices of early performance measured in this study, only the number of taps to attain a performance level of $25 \%$ deviation was significantly correlated with future performance $(r=0.60)$. However, it is difficult for this index to predict the future performance because the correlation coefficient of 0.60 only corresponds to a coefficient of determination of 0.36 , which is statistically significant but not sufficiently high to be considered "predictive."

Moreover, the extent of the initiation-related activity in the putamen was correlated with future motor performance, but not with our initial motor performance indices. This lack of a significant correlation may have been due to the initial level of behavioral instability being masked to some extent by the intentional component (Scholz and Kelso, 1990; Kelso, 1995; De Luca et al., 2010). These results suggest that initiation-related activity in the putamen includes information about the pattern stability of periodic bimanual movements, which can be described theoretically in the HKB model (Haken et al., 1985). Thus, we successfully evaluated the risk of sudden motor error on ongoing periodic bimanual movements by monitoring initiation-related transient putamen activity.

Because the anterior putamen is connected with the prefrontal and premotor cortices, but not the primary motor cortex (Lehéricy et al., 2004), these results suggest that the transient activity of the anterior putamen is likely to be unrelated to motor output. 
Rather, transient putamen activity may be related to motor programming (Kimura, 1990; Lewis et al., 2004; Haruno and Kawato, 2006; Kraft et al., 2007; Aramaki et al., 2010) or to more general functioning, such as attention (Romo et al., 1992) or intention (Scholz and Kelso, 1990; Kelso, 1995; De Luca et al., 2010), implying that the functional range of this area extends beyond bimanual movements.

In the basal ganglia-thalamocortical circuit, the putamen has been found to disinhibit the thalamus by inhibiting the internal globus pallidus, which facilitates cortical activity via the direct motor pathway (Alexander and Crutcher, 1990). Dysfunction in this circuit causes difficulties in initiating movement, including hesitation associated with gait initiation in Parkinson's disease (Okuma and Yanagisawa, 2008). Our results suggest that more difficult movements transiently activate the anterior putamen to a greater extent than less difficult movements during movement initiation. Moreover, neither the ITI deviation during the initial 10 taps nor the RT of the first tap showed a significant difference between BP and BM modes. Thus, it is likely that the greater transient activity of the anterior putamen during the BP mode facilitated the basal ganglia-thalamocortical circuit, and consequently improved initial motor performance in the BP mode. This is consistent with a recent longitudinal study of Parkinson's disease, which demonstrated that more activity in subcortical areas including the putamen was required to execute the same movement as the disease advanced (Carbon et al., 2007).

\section{References}

Alexander GE, Crutcher MD (1990) Functional architecture of basal ganglia circuits: neural substrates of parallel processing. Trends Neurosci 13:266-271.

Aramaki Y, Honda M, Okada T, Sadato N (2006) Neural correlates of the spontaneous phase transition during bimanual coordination. Cereb Cortex 16:1338-1348.

Aramaki Y, Osu R, Sadato N (2010) Resource-demanding versus costeffective bimanual interaction in the brain. Exp Brain Res 203:407-418.

Brett M, Anton J, Valabregue R, Poline J (2002) Region of interest analysis using an SPM toolbox. Paper presented at the 8th International Conference on Functional Mapping of the Human Brain, Sendai, Japan, June 2-6, 2002.

Carbon M, Felice Ghilardi M, Dhawan V, Eidelberg D (2007) Correlates of movement initiation and velocity in Parkinson's disease: a longitudinal PET study. Neuroimage 34:361-370.

De Luca C, Jantzen KJ, Comani S, Bertollo M, Kelso JA (2010) Striatal activity during intentional switching depends on pattern stability. J Neurosci 30:3167-3174

Eichele T, Debener S, Calhoun VD, Specht K, Engel AK, Hugdahl K, von Cramon DY, Ullsperger M (2008) Prediction of human errors by maladaptive changes in event-related brain networks. Proc Natl Acad Sci U S A 105:6173-6178.

Haken H, Kelso JA, Bunz H (1985) A theoretical model of phase transitions in human hand movements. Biol Cybern 51:347-356.

Haruno M, Kawato M (2006) Different neural correlates of reward expectation and reward expectation error in the putamen and caudate nucleus during stimulus-action-reward association learning. J Neurophysiol 95:948-959.

Kagerer FA, Summers JJ, Semjen A (2003) Instabilities during antiphase bimanual movements: are ipsilateral pathways involved? Exp Brain Res 151:489-500.

Kelso JA (1984) Phase transitions and critical behavior in human bimanual coordination. Am J Physiol 246:R1000-R1004.

Kelso JA (1995) Dynamic patterns: the self-organization of brain and behavior. Cambridge, MA: MIT.

Kelso JA (2010) Instabilities and phase transitions in human brain and behavior. Front Hum Neurosci 4:23.

Kennerley SW, Diedrichsen J, Hazeltine E, Semjen A, Ivry RB (2002) Callosotomy patients exhibit temporal uncoupling during continuous bimanual movements. Nat Neurosci 5:376-381.

Kimura M (1990) Behaviorally contingent property of movement-related activity of the primate putamen. J Neurophysiol 63:1277-1296.

Kraft E, Chen AW, Flaherty AW, Blood AJ, Kwong KK, Jenkins BG (2007) The role of the basal ganglia in bimanual coordination. Brain Res 1151:62-73.

Lehéricy S, Ducros M, Van de Moortele PF, Francois C, Thivard L, Poupon C, Swindale N, Ugurbil K, Kim DS (2004) Diffusion tensor fiber tracking shows distinct corticostriatal circuits in humans. Ann Neurol 55:522-529.

Lewis PA, Wing AM, Pope PA, Praamstra P, Miall RC (2004) Brain activity correlates differentially with increasing temporal complexity of rhythms during initialisation, synchronisation, and continuation phases of paced finger tapping. Neuropsychologia 42:1301-1312.

Mechsner F, Kerzel D, Knoblich G, Prinz W (2001) Perceptual basis of bimanual coordination. Nature 414:69-73.

Meyer-Lindenberg A, Ziemann U, Hajak G, Cohen L, Berman KF (2002) Transitions between dynamical states of differing stability in the human brain. Proc Natl Acad Sci U S A 99:10948-10953.

Monno A, Chardenon A, Temprado JJ, Zanone PG, Laurent M (2000) Effects of attention on phase transitions between bimanual coordination patterns: a behavioral and cost analysis in humans. Neurosci Lett 283:93-96

Müller K, Kleiser R, Mechsner F, Seitz RJ (2009) Perceptual influence on bimanual coordination: an fMRI study. Eur J Neurosci 30:116-124.

Okuma Y, Yanagisawa N (2008) The clinical spectrum of freezing of gait in Parkinson's disease. Mov Disord 23 [Suppl 2]:S426-S430.

Romo R, Scarnati E, Schultz W (1992) Role of primate basal ganglia and frontal cortex in the internal generation of movements. II. Movementrelated activity in the anterior striatum. Exp Brain Res 91:385-395.

Sadato N, Yonekura Y, Waki A, Yamada H, Ishii Y (1997) Role of the supplementary motor area and the right premotor cortex in the coordination of bimanual finger movements. J Neurosci 17:9667-9674.

Scholz JP, Kelso JA (1990) Intentional switching between patterns of bimanual coordination depends on the intrinsic dynamics of the patterns. J Mot Behav 22:98-124.

Semjen A, Summers JJ, Cattaert D (1995) Hand coordination in bimanual circle drawing. J Exp Psychol Hum Percept Perform 21:1139-1157.

Temprado JJ, Monno A, Zanone PG, Kelso JA (2002) Attentional demands reflect learning-induced alterations of bimanual coordination dynamics. Eur J Neurosci 16:1390-1394.

Weissman DH, Roberts KC, Visscher KM, Woldorff MG (2006) The neural bases of momentary lapses in attention. Nat Neurosci 9:971-978.

Yamanishi J, Kawato M, Suzuki R (1980) Two coupled oscillators as a model for the coordinated finger tapping by both hands. Biol Cybern 37:219225 . 